\title{
Multisite Controlled Blinded Field Studies to Evaluate the Effect of an Oral Live Escherichia Coli Bivalent F4/F18 Vaccine in Pig Nurseries with Mild Cases of Post-Weaning Diarrhea or Subclinical ETEC Infections.
}

Eric Nadeau ( $\square$ nadeau_eric@elanco.com )

Elanco Animal Health Inc https://orcid.org/0000-0001-5817-620X

Danielle Tremblay

Elanco Animal Health Inc

Anna Magdalena Naderer

Klifovet Ag

Klaus Hellmann

Klifovet AG

\section{Research}

Keywords: Pig, PWD, Colibacillosis, Subclinical, Weight, ETEC, F4, F18, Diarrhea, Vaccine

Posted Date: November 18th, 2020

DOI: https://doi.org/10.21203/rs.3.rs-106327/v1

License: (1) This work is licensed under a Creative Commons Attribution 4.0 International License. Read Full License 


\section{Abstract}

Background: Post-weaning diarrhea (PWD) caused by Escherichia coli is a worldwide economically important disease in pigs, linked with increased mortality and weight loss. F4- and F18-enterotoxigenic E. coli (ETEC) are the most diagnosed causes of PWD. The efficacy of live oral E. coli vaccines has been previously demonstrated on farms with confirmed clinical cases of PWD. However, the impact of vaccination in subclinical cases with mild or not noticeable clinical manifestations needed to be assessed. This study evaluated the effect of an oral dose of a live $E$. coli bivalent F4/F18 vaccine in nurseries without ongoing clinical PWD outbreaks but with a history of pathogenic $E$. coli circulating within the herd. Two controlled blinded studies were performed under commercial conditions in a total of 6 European sites. Overall, 1941 vaccinated piglets and 1976 control piglets were enrolled and were monitored for health, growth, and feed conversion rate.

Results: No clinical outbreak of ETEC PWD has been observed in the tested farms. Two farms faced episodes of non-ETEC enteric diseases that required treatments. Nursery weight gain was not statistically different between groups in these two farms. However, the nursery weight gain of the vaccinated pigs was significantly higher by $0.80,0.87$ and $1.24 \mathrm{~kg}$ than of the control pigs in three of the four farms without non-ETEC enteric diseases $(p<0.05)$ and by $0.69 \mathrm{~kg}(p=0.051)$ in the fourth one, leading to numerically heavier pigs at the exit of the four nurseries. No statistically difference was observed for the feed conversion rate, though it was numerically lower for vaccinated pigs of the two tested farms.

Conclusions: Recent European legislations aimed at reducing the use of antibiotics and banning therapeutic use of zinc oxide in feed are leading to a search for alternatives to compensate animal health impacts and economic losses due to PWD. These studies demonstrate that, even in the absence of convincing clinical signs of PWD, ETEC infections limit nursery pig optimal growth performance that can be mitigated by vaccination with an oral live E. coli bivalent F4/F18 vaccine. These findings corroborate previous studies showing the effect of vaccination in clinical outbreaks of PWD.

\section{Background}

Escherichia coli (E. coli) related post-weaning diarrhea (PWD) is a worldwide economically important disease in pigs due to increased mortality, weight loss and heterogeneity, slow growth, and additional treatment costs (Luppi et al., 2016; Lyutskanov, 2011; Fairbrother and Nadeau, 2010; Svensmark et al., 1989). PWD is mainly caused by enterotoxigenic E. coli (ETEC) that express F4 (K88) or F18 adhesive fimbriae mediating the colonization of the intestinal mucosa and producing enterotoxins that induce secretory diarrhea (Fairbrother and Nadeau, 2019). PWD is typically described as a disease occurring in the first weeks after weaning characterized by sudden death or by diarrhea, dehydration, marked reduction in feed consumption and growth retardation in surviving piglets (Fairbrother and Nadeau, 2019; Rhouma et al., 2017). Additionally, ETEC infections with evident colonization of the pig intestine but without noticeable diarrhea and mortality were sparsely reported and were associated with lower growth performance after weaning (Pollock et al., 2019; Athanasiadou et al., 2010; Hampson, 1994).

For decades, antibiotics, particularly colistin, in combination with therapeutic levels of zinc oxide in the feed were widely used to control PWD across Europe and globally. The emergence of antimicrobial resistance among E. coli isolates (Rhouma et al., 2017; Jahanbakhsh et al., 2016; Luppi et al., 2015) and the recent European adopted measures to significantly reduce the use of colistin in animals (EMA, 2016) and to ban the therapeutic use of zinc oxide in feed (EMA, 2017) highlight the need for alternative control strategies.

An oral live E. coli bivalent F4/F18 vaccine against PWD caused by F4- and/or F18-ETEC (Coliprotec ${ }^{\circledR}$ F4/F18, Prevtec Microbia $\mathrm{GmbH}$, Germany) is available in Canada and the European Union. It has been demonstrated that the use of this oral live $E$. coli vaccine, both in farms with confirmed clinical cases of PWD and in controlled ETEC challenge studies, improved the health of pigs and reduced ETEC intestinal colonization, ETEC fecal shedding, need for antibiotic treatments and weight loss due to F4- and/or F18-ETEC infection (Meymerit et al., 2019; Gibellini and Perro, 2019; Fily et al., 2019; Vangroenwegue and Bonny, 2018; Vangroenwegue and De Braekeleer, 2018; Vangroenwegue et al., 2018; Piqué et al., 2018; Vangroenwegue, 2017; Sánchez Uribe et al., 2017; Nadeau et al., 2017; Gibellini et al., 2017; Fairbrother et al., 2017; Nadeau et al., 2016). Hence, oral vaccination of pigs with a live E. coli vaccine against F4- and F18-ETEC represents a sustainable, practical, and effective approach in such clinical situations. 
The impact of vaccination of herds with subclinical PWD cases, where the load of ETEC in the pig intestine is low, resulting in mild clinical outcomes or not clinically noticeable cases, needed to be assessed. This study aimed to evaluate the effect of an oral live E. coli bivalent F4/F18 vaccine in pig nurseries without major outbreaks of PWD but with a history of pathogenic E. coli circulating within the herd. The hypothesis was that although the pigs appear healthy, the subclinical ETEC infection impairs their growth performance and vaccination with an oral live E. coli bivalent F4/F18 vaccine would reduce the negative impact like weight loss, slow growth and weight heterogeneity due to the ETEC presence in the intestine.

\section{Methods}

\section{Animals and trial design}

Two controlled, randomized, blocked and blinded clinical field studies were performed under commercial conditions at multiple sites in Europe according to Good Clinical Practice (GCP VICH GL9). The studies compared a group of piglets vaccinated with one oral dose of the live E. coli bivalent F4/F18 vaccine (Coliprotec ${ }^{\circledR}$ F4/F18, Prevtec Microbia GmbH, Germany) with a non-vaccinated control group of piglets. The vaccine was prepared with a solution of $5 \mathrm{~g}$ of skimmed milk powder per liter of water; the control piglets received the skimmed milk solution only. The skimmed milk was used to mask the treatment and to neutralize chlorine or other sanitizers that could be present in the water. Pigs were monitored until the end of the nursery period (flatdeck). Nurseries refer to facilities that house newly weaned pigs until they reach the grower/finisher stage.

The first study, performed in 2017, included one production batch for each of the 3 German farms tested (sites GA, GB and GC). The second study took place between 2016 and 2018 and comprised 2 German farms (sites GD and GE) and one Danish farm (site DA). Two consecutive production batches were enrolled for GD and DA. The trial was stopped after one production batch in GE due to an outbreak of porcine parvovirosis. Details of study sites are presented in the Table 1. All selected farms were considered at risk of mild E. coli episodes based on historical data of the previous 6 months. This risk was more obvious on GC for which cases of PWD characterized by low prevalence of pigs showing mild diarrhea due to F4-ETEC were diagnosed in weaned batches prior the study.

The studies were blinded at each site by using separate teams to administer the vaccine/control product and to perform clinical examinations. Procedures were in place to avoid cross-vaccination between groups: separate access to the production units, specific clothes, gloves and shoes that were changed between groups, and dedicated equipment whenever possible, otherwise the equipment was thoroughly cleaned and disinfected between groups.

The number of pigs enrolled per study site and production batch are presented in Table 1. Inclusion criteria comprised piglets derived from sows not vaccinated against $E$. coli, physically healthy and at least 18 days of age at the day of vaccination (day 0 ). Animals moribund at day 0 or that received antimicrobials active against $E$. coli from day -3 to day 0 were not included into the study. A total of 2242 piglets ( 1109 vaccinated and 1133 control) and 1675 piglets (832 vaccinated and 843 control) were enrolled at day $-1 \pm 1$ for the first and second study, respectively.

The experimental timeline and approximate age of the pigs are presented in Fig. 1. Piglets of GA were vaccinated using bowls at the day of weaning (aged $20 \pm 1$ days). Dosing on GB and GC was done in the farrowing unit by oral drenching at a similar age as GA's piglets and were weaned at approximately 28 and 25 days, respectively. Pigs of GD, GE and DA were vaccinated in the farrowing unit using bowls at $20 \pm 2$ days of age and then weaned at approximately 24,26 and 30 days of age, respectively (Table 1). Treatment groups of GA were balanced by sex and body weight before treatment on day 0 . For piglets of GB, GC, GD, GE and $\mathrm{DA}$, mother sows, i.e. the complete litter, were allocated to study groups based on a pre-established randomization list as vaccination was done prior to weaning.

The studies terminated at each farm at the end of the nursery period at the study day 48,53 and 42 for GA, GB and GC, and at the study day 48,78 and 50 for the sites GD, GE and DA (Table 1 and Fig. 1). 
Table 1

Details of the study sites and number of pigs enrolled

\begin{tabular}{|c|c|c|c|c|c|c|c|c|c|c|c|}
\hline Study & $\begin{array}{l}\text { Study } \\
\text { site }\end{array}$ & Location & $\begin{array}{l}\text { Type } \\
\text { of } \\
\text { farm } 1\end{array}$ & $\begin{array}{l}\text { No. } \\
\text { of } \\
\text { sows }\end{array}$ & $\begin{array}{l}\text { No. of } \\
\text { nursery } \\
\text { places }\end{array}$ & $\begin{array}{l}\text { No. } \\
\text { of } \\
\text { pigs } \\
\text { per } \\
\text { unit }\end{array}$ & $\begin{array}{l}\text { Pig } \\
\text { genetic } \\
\text { line }\end{array}$ & $\begin{array}{l}\text { Weaning } \\
\text { age ( } \\
\text { days of } \\
\text { life) }\end{array}$ & $\begin{array}{l}\text { Study } \\
\text { length } \\
\text { (days) }\end{array}$ & $\begin{array}{l}\text { Vaccinated } \\
\text { pigs } \\
\text { (n) }\end{array}$ & $\begin{array}{l}\text { Control } \\
\text { pigs } \\
\text { (n) }\end{array}$ \\
\hline \multirow[t]{3}{*}{ First } & $\mathrm{GA}$ & $\begin{array}{l}\text { North } \\
\text { Germany }\end{array}$ & $\mathrm{FN}$ & 550 & 2700 & 32 & $\mathrm{BHZP}$ & 21 & 48 & 383 & 382 \\
\hline & GB & $\begin{array}{l}\text { South } \\
\text { Germany }\end{array}$ & $\mathrm{FF}$ & 450 & 2100 & 100 & $\begin{array}{l}\text { Danish } \\
\text { Landrace }\end{array}$ & $26-28$ & 53 & 361 & 362 \\
\hline & GC & $\begin{array}{l}\text { South } \\
\text { Germany }\end{array}$ & $\mathrm{FN}$ & 500 & 3000 & 40 & $\begin{array}{l}\text { Danish } \\
\text { Landrace }\end{array}$ & 25 & 42 & 365 & 389 \\
\hline \multirow[t]{5}{*}{ Second } & \multirow[t]{2}{*}{ GD } & \multirow[t]{2}{*}{$\begin{array}{l}\text { North } \\
\text { Germany }\end{array}$} & \multirow[t]{2}{*}{ FF } & \multirow[t]{2}{*}{260} & \multirow[t]{2}{*}{1200} & \multirow[t]{2}{*}{200} & \multirow[t]{2}{*}{$\begin{array}{l}\text { Topigs } \\
\text { and } \\
\text { Pietrain }\end{array}$} & \multirow[t]{2}{*}{24} & \multirow[t]{2}{*}{48} & $\begin{array}{l}\text { Batch 1: } \\
209\end{array}$ & $\begin{array}{l}\text { Batch } \\
1: 213\end{array}$ \\
\hline & & & & & & & & & & $\begin{array}{l}\text { Batch 2: } \\
226\end{array}$ & $\begin{array}{l}\text { Batch } \\
\text { 2: } 229\end{array}$ \\
\hline & GE & $\begin{array}{l}\text { South } \\
\text { Germany }\end{array}$ & $\mathrm{FF}$ & 100 & 560 & 42 & $\begin{array}{l}\text { DL and } \\
\text { Pietrain } x \\
\text { Duroc }\end{array}$ & 26 & 78 & 97 & 93 \\
\hline & \multirow[t]{2}{*}{ DA } & \multirow[t]{2}{*}{ Denmark } & \multirow[t]{2}{*}{$\mathrm{FN}$} & \multirow[t]{2}{*}{700} & \multirow[t]{2}{*}{1200} & \multirow[t]{2}{*}{ NA } & \multirow{2}{*}{$\begin{array}{l}\text { Duroc, } \\
\text { Danish } \\
\text { Landrace, } \\
\text { Danish } \\
\text { Yorkshire }\end{array}$} & \multirow[t]{2}{*}{30} & \multirow[t]{2}{*}{50} & $\begin{array}{l}\text { Batch 1: } \\
149\end{array}$ & $\begin{array}{l}\text { Batch } \\
1: 153\end{array}$ \\
\hline & & & & & & & & & & $\begin{array}{l}\text { Batch 2: } \\
151\end{array}$ & $\begin{array}{l}\text { Batch } \\
\text { 2: } 155\end{array}$ \\
\hline
\end{tabular}

BHZP: German National Hybrid Breeding Program; NA: Not available; n: Number of enrolled pigs; No.: Approximate number; days: Approximative number of days

Experimental timeline in the 6 tested nursery sites GA, GB, GC, GD, GE and DA. Vaccination, represented as an asterisk, was performed at approximately 21 days of age in all sites (study day 0 ). The white triangle and the black triangle represent the perivaccination weight and the exit weight, respectively. The grey vertical rectangle is the weaning. The scale is the approximate age of the pigs. Numbers in parentheses are the study day.

\section{Preparation And Administration Of Vaccine And Control Products}

Water supply was withheld for approximately two hours prior to the planned bowl vaccination in GA to stimulate drinking of the vaccine/control suspension. The live $E$. coli bivalent F4/F18 vaccine was prepared as per the Summary Product Characteristics (SPC) recommendations for vaccination of each pig with a single oral dose via drinking water systems and consumed within 4 hours after preparation. Briefly, the vials were reconstituted and then diluted with a $5-\mathrm{g} / \mathrm{L} \mathrm{skimmed} \mathrm{milk} \mathrm{solution.} \mathrm{The} \mathrm{skimmed}$ milk solution was used as a stabilizer and for blinding purpose. The required volume per pen was calculated and prepared according to the results of a 4-hour consumption pretest and to the average piglet live body weight. Each dose was diluted to get $25 \mathrm{~mL}$ of the vaccine suspension per $\mathrm{kg}$ live weight. The same amount of the skimmed milk powder solution was administered to the control piglets, based on their body weight. Animals of GB and GC were vaccinated using automatic drenchers administering a single oral dose of $2 \mathrm{~mL}$ per pig after being reconstituted and diluted according to the SPC recommendations using a skimmed milk powder solution $(5 \mathrm{~g} / \mathrm{l})$. Control pigs received $2 \mathrm{~mL}$ orally of the skimmed milk powder solution only. Piglet vaccination at GD, $\mathrm{GE}$ and DA was done pre-weaning using bowls. The live $E$. coli bivalent F4/F18 vaccine and the control treatment were prepared as described for GA. 


\section{Health Observations}

Health observations were performed daily by the farm personnel. The severity of diarrhea was scored as 1 (mild), 2 (moderate) and 3 (severe).

\section{Weight, Daily Weight Gain And Feed Conversion Rate}

Pigs were individually weighted at study day $-1 \pm 1$ (GA: -2, GB: $-2, \mathrm{GC}: 0, \mathrm{GD}:-1, \mathrm{GE}: 0, \mathrm{DA}:-1)$ and then at the end of the nursery. Average weight gain (WG) and average daily weight gain (DWG) were compared between groups from days $-1 \pm 1$ to end of nursery.

The feed intake (FI) was evaluated on GA (individual pen level) and GC (two pens combined) using data available electronically by the automated feeding system. The feed conversion rate (FCR) was calculated by dividing the average daily FI by the arithmetic mean of the average DWG of animals within the respective experimental unit (individual pen for GA and combined pens for GC). No FI measurement was available for the other sites.

\section{Statistics And Analysis Populations}

The observation unit for WG and DWG was the individual animal. For each study, these parameters were analyzed for pigs that completed the study, first for each individual site and then for all sites commingled. The observation unit for FI and FCR were the individual pen for GA and combined two pens for GC based on the technical equipment of the farms. Groups were compared using the Wilcoxon Mann-Whitney test (SAS ${ }^{\circledR}$ version 9.3). A $5 \%$ level of significance $(P<0.05$ for two-sided tests) was used to assess statistical differences.

\section{Results}

\section{General Health, Mortality And Reason For Non-completion}

Study 1: Mild transient diarrhea was observed in two pens of the GA control group for a total of $67(18.2 \%)$ pigs. These were not treated and recovered within 24 hours. In GC only 8 animals (1.1\%) showed mild diarrhea. On the other hand, a major outbreak of diarrhea caused by a combination of Salmonella Typhimurium and E. coli (F18:Sta:Stx2e) occurred in GB from 28 days postvaccination, which required treatment of all animals of both groups with colistin.

Overall, 61 vaccinated (5.5\%) and 40 control (3.5\%) animals did not complete the study 1 . Among these, $28(2.5 \%)$ vaccinated and $20(1.8 \%)$ control pigs were removed due to protocol non-compliance. Twenty-one (1.9\%) vaccinated pigs (GA: 7, GB: 8, GC: 6) and $17(1.5 \%)$ control pigs (GA: 5, GB: 8, GC: 4) were found dead or were euthanized for ethical reasons. Clinical signs and postmortem analyses associated the death to various causes, each observed in only few animals, such as pneumonia, lameness, crushed piglets, diarrhea, meningitis, and arthritis.

Study 2: Mild diarrhea was observed for a few pigs or in a few pens in all 3 sites. An outbreak of respiratory disease (influenza with bacterial infections) in GD resulted in the treatment of all animals about one-week post-vaccination with tiamulin hydrogen fumarate, tulathromycin and metamizole. E. coli edema disease was clinically observed and confirmed with isolation of an F18:Stx2e E. coli strain in samples collected at farm GE. However, at this farm no further batch was included due to a severe outbreak of porcine parvovirus after completion of the first batch. Finally, no other health problem than mild diarrhea for a few pigs was observed in DA.

Overall, a total of 20 (2.1\%) vaccinated (GD: 4, GE: 8, DA: 8) and 21 (2.5\%) control (GD: 8, GE: 6, DA: 7) animals did not complete the study 2. All these pigs were found dead or euthanized for ethical reasons. Like the first study, the death was associated to various causes observed each in only few animals such as pneumonia, lameness, crushed piglet, diarrhea, meningitis and arthritis.

There were no adverse events observed in any of the sites related to the study product. 


\section{Weight Gain And Daily Weight Gain}

Body weight and WG are presented in Table 2. During the nursery period, WG of vaccinated pigs was $0.80 \mathrm{~kg}$ to $1.24 \mathrm{~kg}$ significantly higher $(P<0.05)$ than the WG of the control pigs for $G A, G C$, and $G D$ and $0.69 \mathrm{~kg}$ higher for $D A(P=0.051)$. This difference resulted in heavier vaccinated pigs at the end of the nursery than control pigs, with differences ranging in average from $0.45 \mathrm{~kg}$ to $1.24 \mathrm{~kg}$ per animal. For sites GB and GE, occurrence of non-ETEC enteric infections described above affected the outcome of the studies; thus, no statistically significant difference between vaccinated and control pigs in WG being observed. When combining the data of all sites, WG was significantly higher for vaccinated pigs than control pigs: in average $0.56 \mathrm{~kg}(P=$ $0.038)$ and $0.88 \mathrm{~kg}(\mathrm{p}<0.001)$ more for the first and second study, respectively.

Similar results were observed for DWG, which was significantly higher for vaccinated pigs compared to control pigs for GA (by $16 \mathrm{~g}$ ), GC (by $20 \mathrm{~g}$ ) and GD (by $25 \mathrm{~g}$ ) and numerically higher, though close to significance ( $\mathrm{P}=0.051$ ), for DA (by $14 \mathrm{~g}$ ) (Table 3 and Fig. 2). When data of the respective studies are combined, a significant difference of $13 \mathrm{~g}$ (study $1 ; P=0.028)$ and $17 \mathrm{~g}$ (study $2 ; \mathrm{P}$ $=0.001$ ) were observed in favor of vaccinated pigs. DWG was not significantly different for GB and GE where concomitant nonETEC enteric outbreaks occurred.

Table 2

Body weight and weight gain (WG) for the nursery period

\begin{tabular}{|c|c|c|c|c|c|c|c|c|c|c|}
\hline \multirow[t]{2}{*}{ Study } & \multirow[t]{2}{*}{ Site } & \multicolumn{2}{|l|}{$\mathrm{n}$} & \multicolumn{2}{|c|}{ Start weight (kg) } & \multicolumn{2}{|c|}{ End weight (kg) } & \multicolumn{2}{|l|}{ WG (kg) } & \multirow{2}{*}{$\begin{array}{l}\text { P- } \\
\text { value }^{2}\end{array}$} \\
\hline & & $\begin{array}{l}\text { Vaccinated } \\
\text { pigs }^{1}\end{array}$ & $\begin{array}{l}\text { Control } \\
\text { pigs }^{1}\end{array}$ & $\begin{array}{l}\text { Vaccinated } \\
\text { pigs }\end{array}$ & $\begin{array}{l}\text { Control } \\
\text { pigs }\end{array}$ & $\begin{array}{l}\text { Vaccinated } \\
\text { pigs }\end{array}$ & $\begin{array}{l}\text { Control } \\
\text { pigs }\end{array}$ & $\begin{array}{l}\text { Vaccinated } \\
\text { pigs }\end{array}$ & $\begin{array}{l}\text { Control } \\
\text { pigs }\end{array}$ & \\
\hline \multirow[t]{4}{*}{ First } & GA & 371 & 369 & 5.30 & 5.30 & 28.99 & 28.19 & 23.69 & 22.89 & 0.033 \\
\hline & GB & 319 & 342 & 4.29 & 4.59 & 17.71 & 18.48 & 13.42 & 13.89 & 0.161 \\
\hline & $\mathrm{GC}$ & 358 & 382 & 5.28 & 5.70 & 22.69 & 22.24 & 17.41 & 16.54 & 0.035 \\
\hline & $\begin{array}{l}\text { All } \\
\text { sites }\end{array}$ & 1048 & 1093 & 4.99 & 5.22 & 23.40 & 23.07 & 18.42 & 17.86 & 0.038 \\
\hline \multirow[t]{4}{*}{ Second } & GD & 431 & 434 & 5.36 & 5.37 & 24.13 & 22.89 & 18.77 & 17.53 & $<.001$ \\
\hline & GE & 89 & 87 & 5.97 & 5.99 & 32.29 & 32.80 & 26.32 & 26.81 & 0.840 \\
\hline & DA & 292 & 301 & 4.84 & 5.08 & 25.78 & 25.33 & 20.94 & 20.25 & 0.051 \\
\hline & $\begin{array}{l}\text { All } \\
\text { sites }\end{array}$ & 812 & 822 & 5.24 & 5.33 & 25.62 & 24.83 & 20.38 & 19.50 & $<.001$ \\
\hline \multicolumn{11}{|c|}{$\begin{array}{l}{ }^{1} \text { Vaccinated pigs received one oral dose of Coliprotec F4/F18 vaccine prepared in a } 5 \mathrm{~g} / \mathrm{L} \text { skimmed milk solution either via } \\
\text { bowl or by drenching at } 20 \pm 1 \text { days of age; control pigs received the skimmed milk solution only, via the same route. }\end{array}$} \\
\hline
\end{tabular}

$\mathrm{n}$ : Number of pigs that completed the study; Kg: kilogram 
Average daily weight gain (DWG) for the nursery period

\begin{tabular}{|c|c|c|c|c|c|c|}
\hline \multirow[t]{3}{*}{ Study } & \multirow[t]{3}{*}{ Site } & \multicolumn{2}{|l|}{$\mathrm{n}$} & \multicolumn{2}{|l|}{ DWG (g) } & \multirow[t]{3}{*}{ P-value 2} \\
\hline & & Vaccinated pigs ${ }^{1}$ & & Vaccinated pigs & Control & \\
\hline & & & pigs $^{1}$ & & pigs & \\
\hline \multirow[t]{4}{*}{ First } & GA & 371 & 369 & 474 & 458 & 0.034 \\
\hline & GB & 319 & 342 & 244 & 253 & 0.166 \\
\hline & GC & 358 & 382 & 414 & 394 & 0.036 \\
\hline & All sites & 1048 & 1093 & 384 & 371 & 0.028 \\
\hline \multirow[t]{4}{*}{ Second } & GD & 431 & 434 & 383 & 358 & $<0.001$ \\
\hline & GE & 89 & 87 & 337 & 344 & 0.840 \\
\hline & DA & 292 & 301 & 411 & 397 & 0.051 \\
\hline & All sites & 812 & 822 & 388 & 371 & 0.001 \\
\hline \multicolumn{7}{|c|}{$\begin{array}{l}{ }^{1} \text { Vaccinated pigs received one oral dose of Coliprotec F4/F18 prepared in a } 5 \mathrm{~g} / \mathrm{L} \text { skimmed milk solution either in bowl or by } \\
\text { drenching at } 20 \pm 1 \text { days of age; control pigs received the skimmed milk solution only, via the same route. }\end{array}$} \\
\hline
\end{tabular}

n: Number of pigs that completed the study; g: gram

\section{Average Daily Feed Intake And Feed Conversion Rate}

No statistically difference was observed for the FI and FCR for GA, GC and when data of both sites are combined, though the FCR was numerically lower for vaccinated pigs than for control pigs for the analyses of the different populations.

Table 4

Average daily feed intake (FI) and feed conversion rate (FCR) for the nursery period of the study sites GA and GC

\begin{tabular}{|c|c|c|c|c|c|c|c|}
\hline \multirow[t]{2}{*}{ Site } & \multicolumn{2}{|l|}{$\mathrm{n}$} & \multicolumn{2}{|l|}{$\mathrm{FI}(\mathrm{g})$} & \multicolumn{2}{|l|}{ FCR } & \multirow[t]{2}{*}{ P-value ${ }^{2}$} \\
\hline & Vaccinated pigs $^{1}$ & $\begin{array}{l}\text { Control } \\
\text { pigs }^{1}\end{array}$ & Vaccinated pigs & $\begin{array}{l}\text { Control } \\
\text { pigs }\end{array}$ & Vaccinated pigs & $\begin{array}{l}\text { Control } \\
\text { pigs }\end{array}$ & \\
\hline GA & 12 & 11 & 703 & 705 & 1.48 & 1.53 & 0.131 \\
\hline GB & 5 & 4 & 673 & 672 & 1.63 & 1.66 & 0.906 \\
\hline GC & 17 & 15 & 694 & 695 & 1.53 & 1.57 & 0.141 \\
\hline
\end{tabular}

$\mathrm{n}$ : Number of pen (GA) or combined 2 pens (GC)

\section{Discussion}

The results of this study show a positive effect of vaccinating pigs with an oral live $E$. coli bivalent F4/F18 vaccine in farms facing negligible clinical signs related to $E$. coli infections after weaning. The vaccination resulted in significantly higher weight gain 
compared to non-vaccinated pigs. Results from reported field trials and laboratory challenge assays confirmed that vaccination with such PWD oral live E. coli vaccines improved the health of pigs and reduced ETEC intestinal colonization, ETEC fecal shedding, use of antibiotics and weight loss due to either F4-ETEC and/or F18-ETEC (Meymerit et al., 2019; Gibellini and Perro, 2019; Fily et al., 2019; Vangroenwegue and Bonny, 2018; Vangroenwegue and De Braekeleer, 2018; Vangroenwegue et al., 2018; Piqué et al., 2018; Vangroenwegue, 2017; Sánchez Uribe et al., 2017; Nadeau et al., 2017; Gibellini et al., 2017; Fairbrother et al., 2017; Nadeau et al., 2016). These previous studies were performed in proven cases of PWD associated with significant clinical outcomes such as death, diarrhea and weight loss. However, there was a need to assess the impact of vaccination of herds where the load of pathogenic E. coli is low resulting in mild clinical signs or not clinically noticeable by the producer or the veterinarian. The latter can be referred to as subclinical PWD.

Four out of the six tested farms (GA and GC of the first study; GD and DA of the second study) had no major outbreak of concomitant enteric diseases. In those farms, ETEC problems were mild or not clinically noticeable during the study as demonstrated by the small number of dead or euthanized pigs, the non-ETEC confirmation in samples taken from death/euthanasia of these animals and finally, the small number of animals with marked diarrhea. Mild transient diarrhea was observed in $19 \%$ of the GA control pigs. However, no E. coli diagnosis was performed since the diarrhea was very mild and pigs recovered in about 24 hours without treatment. The fact that only control pigs suffered from this mild transient diarrhea suggests a protective effect of the vaccination against F4- and/or F18-ETEC strains in the herd, though other causes cannot be excluded. On this farm, vaccination resulted to $0.8 \mathrm{~kg}$ improved nursery WG for vaccinated piglets compared to control animals. Regarding the site GC, diagnostics done in PWD cases in weaned batches prior to the study identified F4:STa:STb as the cause and confirmed that the site was at high risk of ETEC infections. The same site experienced similar diarrhea episodes after the study, also caused by the F4:STa:STb strain, but in addition an F18:STa:STb strain. Interestingly, only 8 animals had mild diarrhea and no pathogenic E. coli strains were detected during the study, confirming the absence of a PWD outbreak on this farm while vaccination study was carried out. It is reported that Coliprotec ${ }^{\circledR}$ F4/F18 reduces the fecal shedding of F4-ETEC and F18-ETEC by infected pigs (Nadeau et al., 2017). Therefore, vaccination of half of the litters may have reduced the ETEC bacterial load of the herd to a level limiting the occurrence of ETEC-related clinical signs after weaning, but without eradicating the infection in the control group. This is supported by the fact that the vaccination resulted in higher nursery WG of $0.87 \mathrm{~kg}$ and DWG of $20 \mathrm{~g} / \mathrm{day}$.

The Danish site (DA) showed the lowest risk for ETEC diseases as no recent ETEC problem had been diagnosed before the study started. Furthermore, no diarrhea was observed during the study except for very few animals with transient mild diarrhea. Despite this very low ETEC infection pressure, WG during the nursery period was statistically significantly higher for the vaccinated pigs compared to the control pigs; though it was the lowest difference $(0.69 \mathrm{~kg})$ observed amongst the four farms without concomitant enteric outbreaks.

The site GD was associated with the most important reduction of weight loss by vaccination: vaccinated pigs gained $1.24 \mathrm{~kg}$ more weight $(P<0.001)$ than control pigs by the end of the nursery period. This site faced an influenza episode complicated with bacterial infections that led to the treatment of all pigs with tiamulin, tulathromycin and metamizole. It is possible that the stress caused by the viral infection may have increased the differences in WG between vaccinated and control pigs.

For sites GB and GE, where concomitant clinical enteric diseases have been diagnosed, no difference in weight gain between vaccinated and control pigs was observed. These two farms had overall the lowest nursery DWG amongst the tested farms reflecting the negative impact of the clinical outbreaks of enteric diseases on the health of the pigs, whether the pigs had been vaccinated with the live $E$. coli oral bivalent vaccine or not. Since the protective effect of the oral live $E$. coli bivalent F4/F18 vaccine is related to specific anti-F4 and anti-F18 secreted IgM and IgA (Nadeau et al., 2017), it is expected to have no preventive effect on concomitant enteric diseases such as Salmonella or parvovirus. More studies are needed to determine the impact of concomitant systemic and enteric outbreaks on the effects of the live E. coli oral bivalent vaccine when administered to piglets subjected to subclinical cases of PWD.

Diarrhea caused by ETEC is a result of multiple factors and is clinically observed when the amount of fluid from the small intestines exceeds the colonic capacity for fluid reabsorption. To induce noticeable clinical signs, ETEC strains must attach to specific receptors onto the jejunal and ileal epithelium brush borders and then proliferate while secreting enterotoxins leading to reduction of intestinal absorption and fluid movement into the intestinal lumen (Fairbrother and Nadeau, 2019; Nagy and Fekete,

Page $8 / 15$ 
2005). The most important E. coli enterotoxins in pigs are STa, STb, LT and EAST1 (Fairbrother and Nadeau, 2019). To induce noticeable diarrhea in nursery pigs, large amount of ETEC (at least $10^{9}$ to $10^{10}$ ) colonizing and proliferating in the intestines are required (Sun and Kim, 2017). The degree of colonization and multiplication of ETEC in the intestine determine whether the infection results in clinical signs, including diarrhea. On the other hand, the absence of noticeable diarrhea in a herd does not exclude a negative impact of the ETEC infection on the pig gut health and performance. Challenging healthy susceptible piglets with a pathogenic strain of ETEC may induce severe diarrhea, but it can also induce a subclinical form of PWD (Opapeju et al., 2009). Subclinical infections without noticeable diarrhea or mortality but with marked intestinal colonization following ETEC exposure are reported in the literature for porcine challenge models (Pollock et al., 2019). It is also reported that ETEC colonization at a subclinical level after weaning can negatively affect the growth performance of pigs (Athanasiadou et al., 2010; Hampson, 1994).

E. coli enterotoxins harbor functions beyond fluid and electrolyte hypersecretion that can impair the gut health. As examples, STb is able to alter the tight junction complexes that increases the permeability of the intestinal epithelium and might modulate innate immune responses, while STa enhances the secretion of pro-inflammatory cytokines and chemokines in the small intestinal lumen (Wang et al., 2019.). ETEC infection is also associated with an important microbiota change in the distal small intestines but not in the feces (Pollock et al., 2019). Although diarrhea may not be triggered by an ETEC infection, the enterotoxin functions, other than hypersecretion, may affect the gut health in the jejunum and the ileum, being the major colonization sites for porcine F4-ETEC and F18-ETEC (Fairbrother and Nadeau, 2019) and where a high uptake of nutrients occurs. The impact of subclinical ETEC infections may be exacerbated following weaning when a decrease of digestive and adsorptive capacities of the intestines due to villous atrophy and crypt hyperplasia are observed (Thompson and Friendship, 2019).

Knowing that oral live $E$. coli swine vaccines significantly reduce the ETEC load in the intestine and shedding in the feces (Fairbrother et al., 2017; Nadeau et al., 2017), the higher weight gain observed for vaccinated pigs may be related to a control of this potential impairment of the gut health by the subclinical ETEC infection. However, a direct impact of such live E. coli oral vaccines on gut health cannot be excluded as these vaccine strains colonize the intestines at the peri-weaning moment; when the establishment of the post-weaning intestinal flora is critical. Accordingly, it has been reported that the oral administration of Coliprotec $\circledast$ live bivalent F4/F18 E. coli vaccine just before weaning enables the stabilization of the intestinal microbiota after weaning with the proliferation of short-chain fatty acid (SCFA)-producing bacteria, such as Prevotella sp., known to be beneficial to gut health (Luise et al., 2020). Interestingly, the authors also reported a higher nursery average daily weight gain for the vaccinated pigs than for the non-vaccinated pigs.

The existence of subclinical ETEC infection in pigs is not well described in the literature, though reported by few research groups (Pollock et al, 2019; Athanasiadou et al., 2010; Opapeju et al., 2009; Hampson, 1994). One reason for this is the difficulty to detect ETEC in healthy looking pigs. Confirmation of the presence of ETEC in pigs is based on the bacteriological examination of fecal or intestinal content samples on agar plates, selection of isolated colonies and identification by PCR of genes for the fimbriae and the toxins (Fairbrother and Nadeau, 2019; Luppi et al., 2017). This diagnostic approach is sensitive enough for clinical cases of ETEC for which it is possible to select few piglets in the acute phase of diarrhea; when the ETEC fecal load is high enough to be distinguished on the agar plates from the normal E. coli microbiota. However, in mild or subclinical cases of ETEC infections, it is not possible to select few pigs that are more likely to heavily shed ETEC as they all look healthy. Furthermore, the fact that a weaning batch is composed of either susceptible or resistant pigs for ETEC infection makes the sampling more difficult; the susceptibility being driven by the presence of specific receptors for F4 and F18 fimbriae onto the intestinal epithelium (Luise et al., 2019). The diagnostic approach used for acute ETEC infection is therefore less appropriate for the detection of ETEC in subclinical cases of PWD. It would necessitate high number of sampled pigs and the detection of genes by a fairly sensitive method. Though achievable for study purposes, this approach would be cost-prohibitive for routine diagnostic. One approach may be to sample many pigs on a farm using rectal swabs and then to pool the samples in batches to reduce the number of analyses for the detection of the genes by real-time PCR directly in suspended pooled samples. The validation of such an approach would be required to confirm its suitability to detect subclinical cases of PWD.

The combination of colistin and therapeutic levels of zinc oxide was a widely used metaphylactic practice across the European Union and in many other regions of the world to control PWD. However, several measures to reduce the use of colistin in foodproducing animals have been recently adopted by EMA following the identification of a transferable MCR-1 resistance mechanism 
(EMA, 2016). Colistin is now classified as a Category 2 antibiotics, which means it should be reserved for treating infections in pigs for which no effective alternative treatments exist. In this sense, EU Member States should reduce the use of colistin in pigs at pre-established thresholds without compensation in the use of other categories of antimicrobials. The metaphylactic use of 2400 to $3000 \mathrm{ppm}$ of zinc in the feed is known to reduce diarrhea caused by E. coli, mortality and improve growth of pigs (Luppi, 2017). However, veterinary medicinal products containing zinc oxide will no longer be permitted in the EU from June 2022, following a negative benefit/risk conclusion of EMA's CVMP evaluating the current status of safety and effectiveness (EMA 2017). The already initiated discontinuation of this metaphylactic approach in many European countries opens the search for effective sustainable strategies to control ETEC infections in pig farms. This transitional period is expected to lead to major changes in overall swine gut health, mainly resulting from the trial-and-error approach that could be considered to find alternatives to compensate the economic losses incurred by the withdrawal of the previously used antibiotic products.

Our parallel group blinded studies revealed that mild or subclinical cases of F4- and F18-ETEC infection in pig nurseries can have a negative impact on the growth performance and that pigs orally vaccinated with an oral live $E$. coli bivalent F4/F18 vaccine showed a higher weight gain from about half a kilogram to more than one kilogram per pig on average from the weaning to the end of the nursery period. It is noteworthy that pigs vaccinated peri-weaning maintained a higher weight gain throughout the entire nursery phase in the case of subclinical/mild ETEC infection, likewise described by others in farm trials with confirmed clinical cases of PWD (Meymerit et al., 2019; Gibellini and Perro, 2019; Fily et al., 2019; Vangroenwegue and De Braekeleer, 2018; Vangroenwegue et al., 2018; Piqué et al., 2018). Furthermore, though not statistically different, the FCR was numerically lower for vaccinated pigs than control pigs in both farms where the feed intake was evaluated. The study design limited, however, the assessment of the FCR, particularly due to the low number of experimental units tested and further studies specifically designed for that purpose are needed to make a clear conclusion of the impact of vaccination with this oral live $E$. coli bivalent vaccine for this parameter.

\section{Conclusions}

These current studies demonstrate that, even in the absence of convincing clinical signs of PWD, ETEC infections limit the nursery piglet optimal growth performance and that these effects can be mitigated by vaccination with an oral live E. coli bivalent F4/F18 vaccine. These findings add to studies proving the effect of the vaccine in clinical cases of PWD which clearly demonstrated the efficacy of the oral live bivalent $E$. coli F4/F18 vaccine not only in reducing clinical signs of PWD but also in reducing the negative impact of an ETEC infection on pig growth performance. Further studies are needed to evaluate the importance and the impact of these subclinical ETEC infections in the swine industry and to characterize the effect and the role of the oral live $E$. coli bivalent vaccine in these cases.

\section{Abbreviations}

DWG

Daily weight gain

EAST1

Enteroaggregative heat-stable toxin 1

ETEC

Enterotoxigenic Escherichia coli

F4

Fimbriae F4

F18

Fimbriae F18

FCR

Feed conversion ratio

$\mathrm{FI}$

Feed intake

LT

Page $10 / 15$ 
Heat-labile toxin

PWD

Post-weaning diarrhea

SPC

Summary of Product Characteristics

STa

Heat-stable toxin a

$\mathrm{STb}$

Heat-stable toxin b

Stx2e

Shigatoxin $2 \mathrm{e}$

WG

Weight gain

\section{Declarations}

Ethics approval and consent to participate

- Field trials performed with a veterinary medicinal product approved for use in swine. No additional ethical approval needed. Owner consent to participate was obtained for all owners using an Owner informed consent form following Good Clinical Practices guidelines.

Consent for publication

- Not applicable

Availability of data and materials

- Please contact the author for requests.

Competing interests

- This project was sponsored by Prevtec Microbia. The sponsor made substantial contributions to the study design, conduct of the study, collection, analysis, and interpretation of the data, and the writing of the manuscript. The sponsor of this research is the market authorization holder of the Coliprotec F4/F18 vaccine. EN and DT were, at the time of the study, employees of the sponsor. EN is co-inventor of Coliprotec F4/F18. KH was compensated by the study sponsor for his participation as the principal investigator and study principal monitor while LMN acted as study monitor. EN and DT are current employee of the actual owner and the distributor of Coliprotec F4/F18.

Funding

- These studies were financially supported by Prevtec Microbia, market authorization holder of the Coliprotec $\AA$ F4/F18 vaccine for use in swine at the time of the study.

Authors' contributions

- All authors contributed to the design of the study and interpretation of results. EN, DT and KH managed and supervised the studies. KH and LMN monitored the studies. All authors read and approved the final manuscript.

Acknowledgements

- The pig farmers and veterinarians and all others involved are gratefully acknowledged for their support throughout the study. The authors wish to thank Peter Klein for statistical analyses and Dr. Jutta Verspohl for leading the microbiological analyses. 


\section{References}

Athanasiadou S, Houdijk JG, Eckersall PD, Low JC, Kyriazakis I. Development of infection models to assess subclinical disease in pigs through the use of acute phase proteins as markers. Adv Anim Biosci. 2010;1(1):119.

European Medicines Agency (EMA). Questions and answers on veterinary medicinal products containing zinc oxide to be administered orally to food-producing species Outcome of a referral procedure under Article 35 of Directive 2001/82/EC. EMEA/V/A/118. 2017. https://www.ema.europa.eu/en/documents/referral/zinc-oxide-article-35-referral-questions-answersveterinary-medicinal-products-containing-zinc-oxide_en.pdf. Accessed 27 Sep 2020.

European Medicines Agency (EMA). Updated advice on the use of colistin products in animals within the European Union: development of resistance and possible impact on human and animal health. 2016.

https://www.ema.europa.eu/en/documents/scientific-guideline/updated-advice-use-colistin-products-animals-within-europeanunion-development-resistance-possible_en-0.pdf. Accessed 27 Sep 2020.

Fairbrother JM, Nadeau E. Colibacillosis. In: Zimmerman JJ, Karriker LA, Ramirez A, Schwartz KJ, Stevenson GW, Zhang J, editors. Diseases of Swine. 11th ed. Ames: Blackwell Publishing; 2019. pp. 807-34.

Fairbrother JM, Nadeau E. Colibacillosis. In: Lefèvre PC, Blancou J, Chermette R, Uilenberg G, editors. Infectious and Parasitic Diseases of Livestock. Lavoisier; 2010. p. 917-945.

Fairbrother JM, Nadeau E, Belanger L, Tremblay CL, Tremblay D, Brunelle M, Wolf R, Hellmann K, Hidaldo Á. Immunogenicity and protective efficacy of a single-dose live non-pathogenic Escherichia coli oral vaccine against F4-positive enterotoxigenic Escherichia coli challenge in pigs. Vaccine. 2017;35(2):353-60.

Fily B, Boutin F, Burlot V, Geffroy N, Gin T. Results from 14 farrow-to-finish French farms before and after implementation of Coliprotec $\AA_{F}$ 4/F18 (2019). Proceeding 11th ESPHM Utrecht.

Gibellini M, Perro P. Efficacy of Coliprotec $® F 4 F 18$ in piglets with post-weaning diarrhea due to F18-ETEC in a commercial Italian farm (2019). Proceeding 11th ESPHM Utrecht.

Gibellini M, Ferro P, Scandurra S, Veloci M, Mazzoni C, Scollo A. Efficacy of Coliprotec $®$ F4 in piglets with post-weaning diarrhea due to F4-ETEC in an Italian farm (2017). Proceeding 9th ESPHM Prague.

Hampson DJ. Post-weaning Escherichia coli diarrhea in pigs. In: Gyles CL, editor. Escherichia coli in domestic animals and humans. London: CABl; 1994. pp. 171-91.

Jahanbakhsh J, Smith MG, Kohan-Ghadr HR, Letellier A, Abraham S, Trott DJ, Fairbrother JM. Dynamics of extended-spectrum cephalosporin resistance in pathogenic Escherichia coli isolated from diseased pigs in Québec, Canada. Int J Antimicrob Agents. 2016;48(2):194-202.

Luise D, Spinelli E, Correa F, Salvarani C, Bosi P, Trevisi P. Effects of $E$. coli bivalent vaccine and of host genetic susceptibility to $E$. coli on the growth performance and faecal microbial profile of weaned pigs. Livest Sci. 2020;241:1-8.

Luise D, Lauridsen C, Bosi P, Trevisi P. Methodology and application of Escherichia coli F4 and F18 encoding infection models in post-weaning pigs. J Anim Sci Biotechnol. 2019. doi:10.1186/s40104-019-0352-7.

Luppi A. Swine enteric colibacillosis: diagnosis, therapy and antimicrobial resistance. Porc Health Manag. 2017. doi:10.1186/s40813-017-0063-4.

Luppi A, Bonilauri P, Dottori M, Gherpelli Y, Biasi G, Merialdi G, Maioli G, Martelli P. Antimicrobial resistance of F4 + Escherichia coli isolated from swine in Italy. Transbound Emerg Dis. 2015;62(1):67-71.

Luppi A, Gibellini M, Gin T, Vangroenweghe F, Vandenbroucke V, Bauerfeind R, Bonilauri P, Labarque G, Hidalgo Á. Prevalence of virulence factors in enterotoxigenic Escherichia coli isolated from pigs with post-weaning diarrhoea in Europe. Porc Health Manag.

Page $12 / 15$ 
2016. doi:10.1186/s40813-016-0039-9.

Lyutskanov M. Epidemiological characteristics of post-weaning diarrhoea associated with toxin-producing Escherichia coli in large intensive pig farms. Trakia J Sci. 2011;9(3):68-73.

Meymerit C, Ferreira D, Burlot V, Gin T. Implementation of Coliprotec F4/F18 and a new feed program in a French farrow-to-finish farm to control post-weaning diarrhea and improve performances. 2019. Proceeding 11th ESPHM Utrecht.

Nadeau E, Tremblay D, Bélanger L, Cvejić D, Bauer K, Schneider C, Hellmann K, Hidalgo Á. Field efficacy of Coliprotec® F4, live oral vaccine against post-weaning diarrhoea caused by F4-enterotoxigenic E. coli (F4-ETEC), in German pig farms. 2016. Proceeding 8th ESPHM Dublin.

Nadeau E, Fairbrother JM, Zentek J, Belanger L, Tremblay D, Tremblay CL, Rohe I, Vahjen W, Brunelle M, Hellmann K, Cvejić D, Brunner B, Schneider C, Bauer K, Wolf R, Hidaldo Á. Efficacy of a single oral dose of a live bivalent E. coli vaccine against postweaning diarrhea due to F4 and F18-positive enterotoxigenic E. coli. Vet J. 2017;226:32-9.

Nagy B, Fekete PZ. Enterotoxigenic Escherichia coli in veterinary medicine. Int J Med Microbiol. 2005;295(6-7):443-54.

Opapeju FO, Rademacher M, Payne RL, Krause DO, Nyachoti CM. Inflammation-associated responses in piglets induced with postweaning colibacillosis are influenced by dietary protein level. Livest Sci. 2010;131(1):58-64.

Piqué J, Pérez L, Claver M, Lara M. Investigation of production parameters in a commercial pig farm in Spain with post-weaning diarrhea before and after the implementation of a live non-pathogenic Escherichia coli vaccine (Coliprotec® F4/F18). 2018. Proceeding 10th ESPHM Barcelona.

Pollock J, Hutchings MR, Hutchings KEK, Gally DL, Houdijk JGM. Changes in the ileal, but not fecal, microbiome in response to increased dietary protein level and enterotoxigenic Escherichia coli exposure in pigs. Appl Environ Microbiol. 2019. doi:10.1128/AEM.01252-19.

Rhouma M, Fairbrother JM, Beaudry F, Letellier A. Post weaning diarrhea in pigs: risk factors and non-colistin-based control strategies. Acta Vet Scand. 2017. doi:10.1186/s13028-017-0299-7.

Sánchez Uribe P, Lamrani Á, Núñez P, Hidalgo Á, Martínez J, Oliva JE, Márquez G, Nadeau E. Vaccination against post-weaning diarrhoea with Coliprotec ${ }^{\circledR}$ F4: Production results from a large scale farm in Spain. 2017. Proceeding 9th ESPHM Prague.

Sun Y, Kim SW. Intestinal challenge with enterotoxigenic Escherichia coli in pigs, and nutritional intervention to prevent postweaning diarrhea. Anim Nutr. 2017;3(4):322-30.

Svensmark B, Nielsen K, Willeberg P, Jorsal SE. Epidemiological studies of piglet diarrhea in intensively managed Danish sow herds. II. Post-weaning diarrhea. Acta Vet Scand. 1989;30(1):55-62.

Thompson JR, Friendship RM. Digestive system. In: Zimmerman JJ, Karriker LA, Ramirez A, Schwartz KJ, Stevenson GW, Zhang J, editors. Diseases of Swine. 11th ed. Ames: Blackwell Publishing; 2019. pp. 234-63.

Vangroenwegue F. Application of high energy diets in combination with the Coliprotec $\circledast$ F4 vaccination against post-weaning diarrhea. 2017. Proceeding 9th ESPHM Prague.

Vangroenwegue F, Bonny P. Vaccination with a live bivalent E. coli F4/F18 vaccine for the prevention of F18-ETEC post-weaning diarrhea. 2018. Proceeding 10th ESPHM Barcelona.

Vangroenwegue F, De Braekeleer B. Vaccination with a live bivalent E. coli F4/F18 vaccine results in antibiotic reduction with improved growth and feed conversion rate 2018. Proceeding 10th ESPHM Barcelona.

Vangroenwegue $\mathrm{F}$, Jacobs A, Defoort P. Vaccination with a live bivalent E. coli F4/F18 vaccine for the prevention of F18-ETEC postweaning diarrhea - reduction of mortality and antibiotic use. 2018. Proceeding 10th ESPHM Barcelona. 
Wang H, Zhong Z, Luo Y, Cox E, Devriendt B. Heat-stable enterotoxins of enterotoxigenic Escherichia coli and their impact on host immunity. Toxins. 2019. doi:10.3390/toxins11010024.

\section{Figures}

GA

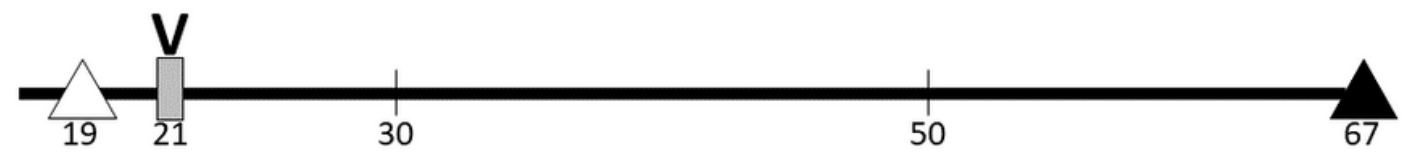

$\mathrm{GB}$

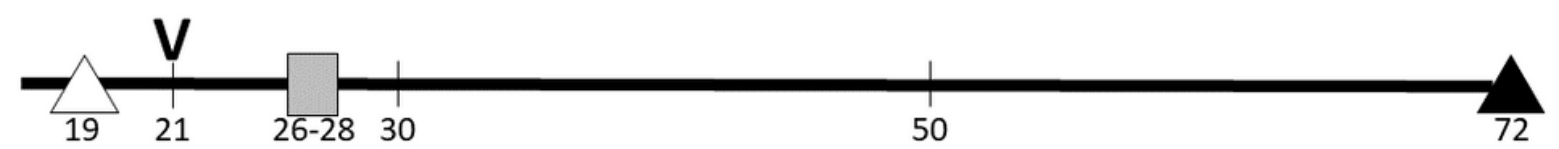

GC

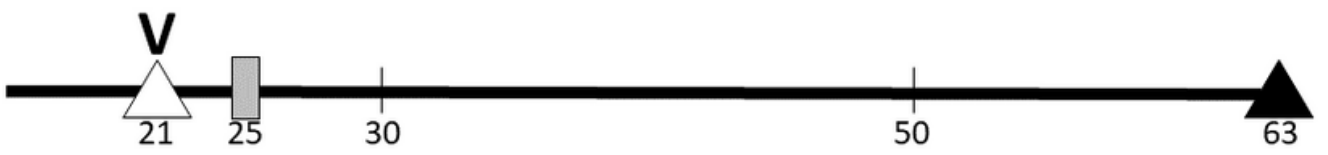

GD

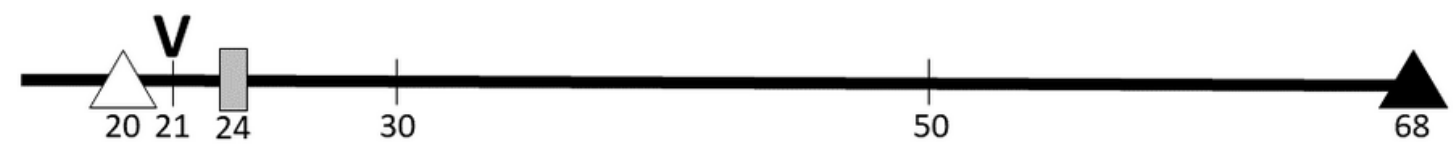

GE

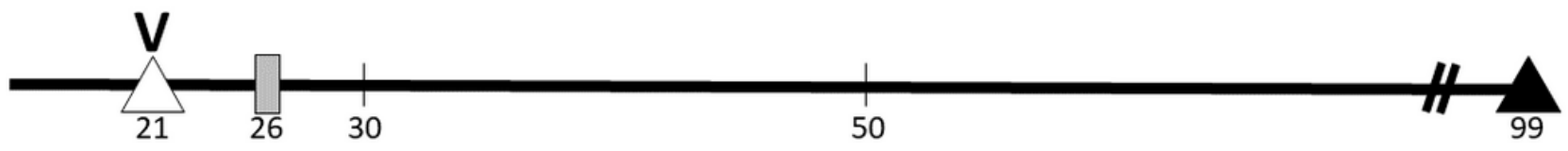

DA

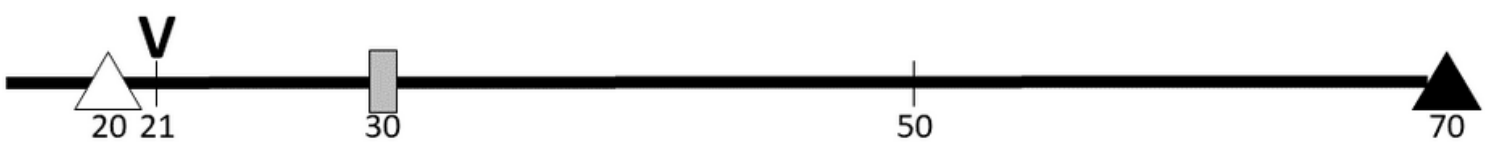

Figure 1

Experimental timeline in the 6 tested nursery sites 

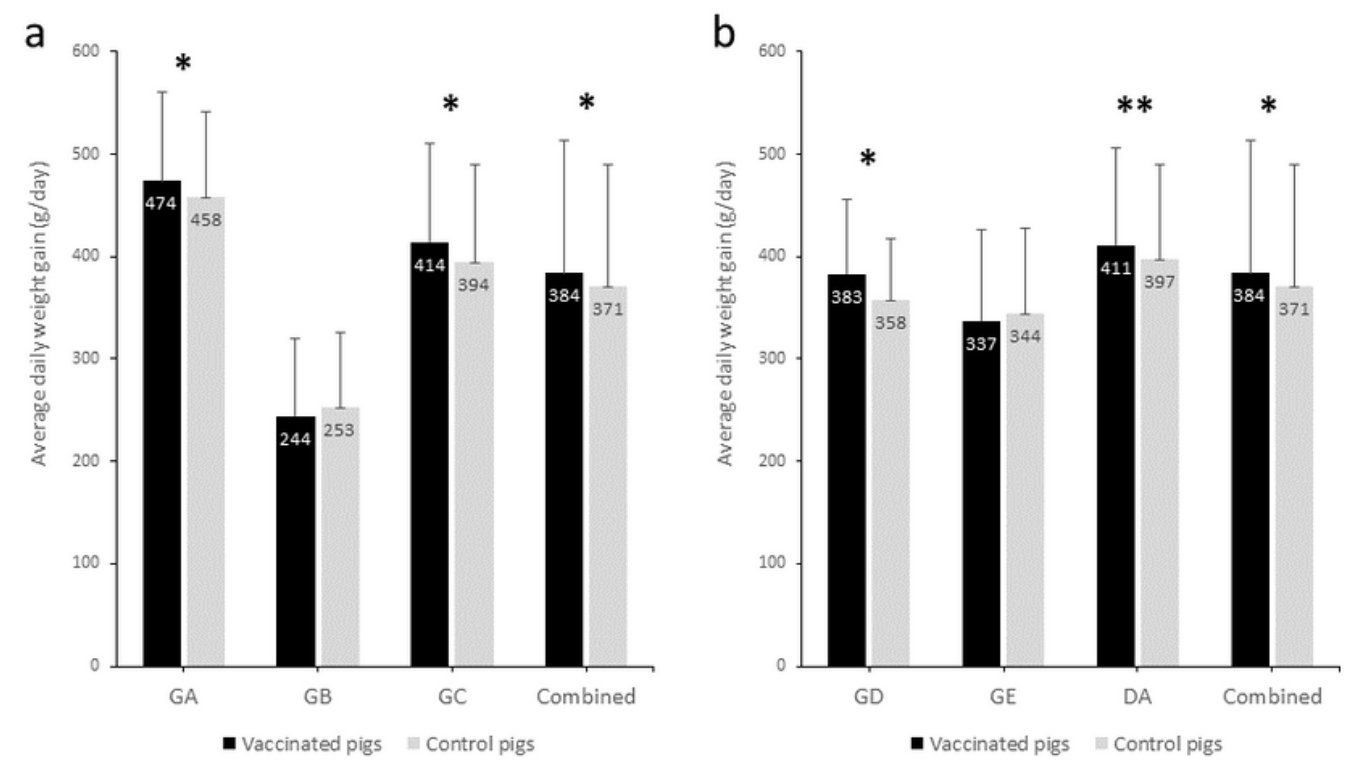

Figure 2

Average daily weight gain (DWG) for the nursery period of the first (a) and second (b) study Comparison of the daily weight gain (DWG) over the total study period (the entire nursery period; study day $-1 \pm 1$ to the end of the nursery) between pigs vaccinated with an oral live E. coli bivalent F4/F18 vaccine and non-vaccinated control pigs. For the first study (a) and the second study (b) results are presented for individual study site and for all study sites combined. Data are reported as the mean and error bars represent the standard error. * Daily weight gain of the vaccinated pigs significantly higher than that of the control pigs $(P<0.05)$. ** $P=0.051$ 